\title{
Recurrent Cytomegalovirus Infection
}

National Cancer Institute

\section{Source}

National Cancer Institute. Recurrent Cytomegalovirus Infection. NCI Thesaurus. Code C156253.

A cytomegalovirus infection that has recurred after a period of remission. 